\title{
A symbolic approach to voltage stability and power sharing in time-varying DC microgrids
}

\author{
Daniele Zonetti, Adnane Saoud, Antoine Girard and Laurent Fribourg
}

\begin{abstract}
In this paper we address the problem of voltage stability and power sharing in DC microgrids with time-varying power demand. By exploiting the monotonicity property enjoyed by the system, and under the assumption of full observability of the bus voltages, we design a centralized, abstractionbased symbolic controller that, once refined into a controller for the original system, ensures the required specifications. Whereas load voltages cannot be measured, we propose an appropriate decomposition of the system, such that the control problem can be reformulated in terms of assume-guarantee contracts to be satisfied by the observable and unobservable components. A constructive procedure to determine suitable contracts is further investigated and the obtained results are validated with two numerical examples.
\end{abstract}

\section{INTRODUCTION}

Direct-current (DC) microgrids have been recognized as a promising choice for the redesign of distribution systems, which are undergoing relevant changes due to the increased penetration of photovoltaic modules, batteries and DC loads [1]. A microgrid is an electrical network gathering a combination of generation units, loads and energy storage elements-in the sequel referred as power units. With the exception of passive loads, all units are interfaced to the DC network through power converters, which are internally controlled to guarantee appropriate power profiles under fast current and voltage perturbations. Some of the units are further equipped with an additional layer of control-termed primary control - which operates at a slower time-scale and guarantees robust and safe operation in presence of large disturbances [2]. From the point of view of the grid then, the individual power units exhibit a highly nonlinear behavior, as they are operated to maintain appropriate power profiles irrespectively from perturbations. The control design is usually addressed by splitting the main control problem in two subproblems. First, establish upper and lower bounds on the power profiles of the individual units that ensure existence of an equilibrium point. Second,

*This research was supported by Labex DigiCosme (project ANR-11LABEX-0045-DIGICOSME) operated by ANR as part of the program "Investissement d'Avenir" Idex Paris Saclay (ANR-11-IDEX-0003-02). This project has received funding from the European Research Council (ERC) under the European Union's Horizon 2020 research and innovation programme (grant agreement No 725144).

D. Zonetti, A. Saoud and L. Fribourg are with Laboratoire Spécification et Vérification, CNRS, ENS Paris-Saclay,61, avenue du Président Wilson, 94235 Cachan Cedex, France. daniele.zonettielsv.fr, fribourgelsv.ens-cachan.fr

A. Saoud and A. Girard are with Laboratoire des Signaux et Systèmes (L2S), CNRS, CentraleSupélec, Université Paris-Sud, Université Paris-Saclay, 3, rue Joliot-Curie, 91192 Gif-sur-Yvette Cedex, France. (adnane.saoud, antoine.girard) @l2s.centralesupelec.fr design a controller that guarantees that such equilibrium voltage is kept sufficiently near the grid nominal value, while achieving an appropriate distribution of power among the units. Both problems received great attention in recent years, see for example [3]-[5] and [6]-[9]. However, the majority of these works assume that power units behave exactly as constant power devices (CPDs) and that the system stability must be inferred by the existence of an asympotically stable equilibrium point of the closed-loop system. Moreover, the control designs do not provide transient guarantees and are usually realized by prioritizing either the verification of the power sharing [6], [7], either the regulation of the voltages near the nominal value [9]. As a result, the fulfillment of both properties can be only guaranteed by the verification of some extra conditions or by the use of additional controllers.

The use of symbolic control techniques has been an ongoing research area in recent years. In such approaches, a symbolic model-a system with a finite number of states and inputsis constructed starting from the original system. When the concrete (original) and abstract (symbolic) systems are linked by some behavioral relation such as simulation, alternating simulation or their approximate versions, a symbolic controller synthesized for the abstraction can be refined into a controller for the original system [10], [11]. As opposed to traditional control theory, which usually focuses on properties such as stability, controllability and tracking of trajectories, symbolic control leverages the use of automata theoretic techniques for the synthesis of controllers that enforce a wide range of specifications such as safety, reachability or more complex objectives such as those expressed in linear temporal logic [12].

In this work, starting from a nonlinear, time-varying model of a DC microgrid, we derive suitable abstractions of the system by exploiting monotonicity and next design symbolic controllers that, once refined into a controller for the original system, ensure the required specifications. We consider two different scenarios. First, we assume that all voltages are measurable. In this case we can construct a monolithic abstraction of the whole system, for which we synthesize a centralized symbolic controller that fulfills the control objectives. Then a sampled-data controller preserving the same features is derived. To deal with the partial information case, we employ a contractbased approach: the DC microgrid is decomposed into two subsystems, each subsystem is assigned an assumeguarantee contract, then a compositionality result ensures 
that if both components satisfy their own contract, the global control specification is achieved. A constructive procedure is further proposed for a systematical exploration of feasible contracts. Although the aforementioned control objectives usually require distributed methods, for simplicity we here only focus on a centralized implementation-these to be intended as a first step towards the development of a more general theory that guarantees the fulfillment of the same objectives in a distributed fashion.

The remainder of the paper is structured as follows. The model of the DC microgrid is presented in Section II and is instrumental to establish the control problem, which is illustrated in Section III. In Section IV then, we show the procedure to derive an abstraction and to design a sampleddata controller that guarantees the control specifications in case of full information. To deal with the partial information case, the contract-based approach is developed in Section V. Theoretical results are validated in Section VI on numerical examples of a two-units and four-units DC microgrid. VI. Conclusions and final remarks follows in Section VII.

Notation All vectors are column vectors. We use $\underline{0}$ to denote matrices of zeros, 1 to denote the vector with all ones and I to denote the identity matrix of appropriate dimensions. $x=\operatorname{col}\left(x_{i}\right)$ denotes a vector with entries $x_{i} \in \mathbb{R}, \operatorname{diag}\left\{a_{i}\right\}$ denotes a diagonal matrix with entries $a_{i} \in \mathbb{R}$. $\mathbb{T}$ is the set of nonnegative reals. Element-wise (Hadamard) division of matrices is denoted by $\oslash \cdot \mathcal{I}_{\delta}(x)$ denotes the ball centered in $x \in \mathbb{R}^{n}$ and of radius $\delta>0$. Given a system $\Sigma$ described by the continuous-time differential inclusion $\dot{x}(t) \in \mathrm{f}(x, u)$, where $x \in \mathcal{X}$ is the state and $u \in \mathcal{U}$ is the input, we will use $\Phi\left(t ; x^{0}, \mathrm{u}\right)$ to denote the set of points reached at time $t \in \mathbb{T}$ from the initial condition $x^{0}$ under the input $\mathrm{u}:[0, t] \rightarrow \mathcal{U}$.

\section{MODELING OF DC MICROGRIDS}

Following the same approach used in [6], we represent a microgrid as a directed graph $\mathcal{G}(\mathcal{N}, \mathcal{E}, \mathcal{B})$, where: $\mathcal{N}$ is the set of nodes, with cardinality $n ; \mathcal{E}$ is the set of edges, with cardinality $t$ and $\mathcal{B} \in \mathbb{R}^{n \times t}$ is the incidence matrix capturing the graph topology. The edges correspond to the transmission lines, while the nodes correspond to the buses where the power units are interfaced. We further define $\mathcal{N}_{S}$ as the subset of nodes associated to controllable power units, i.e. the generation and energy storage units, with cardinality $m$, and $\mathcal{N}_{L}$, as the subset of nodes associated to non-controllable power units, i.e. the loads, with cardinality $n-m$. Accordingly, the collection of voltages and currents at the buses can be partitioned as $V:=\operatorname{col}\left(V_{S}, V_{L}\right) \in \mathbb{R}^{n}$, $I:=\operatorname{col}\left(I_{S}, I_{L}\right) \in \mathbb{R}^{n}$, while the incidence matrix admits the decomposition $\mathcal{B}=\operatorname{col}\left(\mathcal{B}_{S}, \mathcal{B}_{L}\right)$, with $\mathcal{B}_{S} \in \mathbb{R}^{m \times t}$, $\mathcal{B}_{L} \in \mathbb{R}^{(n-m) \times t}$. Since we focus our attention on dominantly resistive transmission lines, it is assumed that each edge associates a weight modeling the conductance $G_{e}>0$ of the line $e \in \mathcal{E}$ and that inductive dynamics are neglected. The interconnection laws between power units are captured by the generalized Ohm's law:

$$
I=\mathcal{L} V
$$

where $\mathcal{L} \in \mathbb{R}^{n \times n}$ is the Laplacian matrix associated to the graph, which is defined as:

$$
\mathcal{L}:=\left[\begin{array}{c}
\mathcal{B}_{S} \\
\mathcal{B}_{L}
\end{array}\right] G\left[\begin{array}{c}
\mathcal{B}_{S} \\
\mathcal{B}_{L}
\end{array}\right]^{\top}=\left[\begin{array}{cc}
\mathcal{L}_{S} & \mathcal{L}_{m} \\
\mathcal{L}_{m}^{\top} & \mathcal{L}_{L}
\end{array}\right] \in \mathbb{R}^{n \times n},
$$

with $G:=\operatorname{diag}\left(G_{e}\right) \in \mathbb{R}^{t \times t}$.

Using the aforementioned partition, we now characterize the dynamics of the power units. With no loss of generality we make the assumption that controllable power units only inject, and not absorb, power and refer to them as sources. Their dynamics is then characterized as follows:

$$
C_{j} \dot{v}_{j}=-G_{j} v_{j}+\frac{u_{j}}{v_{j}}-i_{j}, \quad j \in \mathcal{N}_{S},
$$

with state $v_{j} \in \mathbb{R}$, input $i_{j} \in \mathbb{R}$, constant parameters $C_{j}, G_{j} \in \mathbb{R}$ and a continuous signal $u_{j}: \mathbb{T} \rightarrow \mathcal{P}_{j}$, with $\mathcal{P}_{j}:=\left[\underline{P}_{j}, \bar{P}_{j}\right] \subset \mathbb{R}$. All parameters are positive. These dynamics consist of a lossy capacitive dynamics, with bounded (positive) power injection, interfaced to the grid via a current sink. The term $u_{j}(t)$, denoting the power injection, is usually determined by a primary controller $u_{j}^{\prime}$ via

$$
u_{j}=P_{j}+u_{j}^{\prime}, \quad j \in \mathcal{N}_{S},
$$

with $P_{j} \geq 0$ a nominal reference value. For simplicity, $u_{j}$ is interpreted as a control input.

On the other hand, loads can be described by the dynamics:

$$
C_{k} \dot{v}_{k}=-G_{k} v_{k}+\frac{p_{k}}{v_{k}}-i_{k}, \quad k \in \mathcal{N}_{L},
$$

with state $v_{k} \in \mathbb{R}$, input $i_{k} \in \mathbb{R}$, constant parameters $C_{k}, G_{k}$ and a continuous signal $p_{k}: \mathbb{T} \rightarrow \mathcal{P}_{k}$, with $\mathcal{P}_{k}:=\left[-\underline{P}_{k},-\bar{P}_{k}\right] \subset \mathbb{R}$. All parameters are positive. These dynamics consist of a lossy capacitive dynamics, with bounded time-varying power demand, interfaced to the grid via a current sink. The term $p_{k}(t)$, denoting the (negative) power demand is an unknown signal and thus is interpreted as a time-varying perturbation. Using (II.2), the stacked dynamics of (II.3), (II.4) can be rewritten in compact form:

$$
\left[\begin{array}{l}
C_{S} \dot{V}_{S} \\
C_{L} \dot{V}_{L}
\end{array}\right]=-\left[\begin{array}{cc}
G_{S} & \underline{0} \\
\underline{0} & G_{L}
\end{array}\right]\left[\begin{array}{l}
V_{S} \\
V_{L}
\end{array}\right]+\left[\begin{array}{l}
u \oslash V_{S} \\
p \oslash V_{L}
\end{array}\right]-\left[\begin{array}{c}
I_{S} \\
I_{L}
\end{array}\right]
$$

where

- $V_{S}:=\operatorname{col}\left(v_{j}\right) \in \mathbb{R}^{m}, V_{L}:=\operatorname{col}\left(v_{k}\right) \in \mathbb{R}^{n-m}$ are the state voltages;

- $I_{S}:=\operatorname{col}\left(i_{j}\right) \in \mathbb{R}^{m}, I_{L}:=\operatorname{col}\left(i_{k}\right) \in \mathbb{R}^{n-m}$ are the input currents;

- $u:=\operatorname{col}\left(u_{j}\right) \in \mathcal{P}_{S}$ and $p:=\operatorname{col}\left(P_{k}\right) \in \mathcal{P}_{L}$ are the input and perturbation powers, with

$$
\mathcal{P}_{S}:=\Pi \mathcal{P}_{j} \in \mathbb{R}^{m}, \quad \mathcal{P}_{L}:=\Pi \mathcal{P}_{k} \in \mathbb{R}^{n-m} ;
$$

- $C_{S}:=\operatorname{diag}\left(C_{j}\right), G_{S}:=\operatorname{diag}\left(G_{j}\right), C_{L}:=\operatorname{diag}\left(C_{k}\right)$, $G_{L}:=\operatorname{diag}\left(G_{k}\right)$ are matrices of appropriate dimensions. 
By replacing (II.1)-(II.2) into (II.5) and recalling that $p \in$ $\mathcal{P}_{L}$, the system dynamics can be equivalently described via the following differential inclusion:

$$
\dot{V} \in \mathrm{f}(V, u),
$$

with

$$
\mathrm{f}(V, u):=C^{-1}\left(-(\mathcal{L}+G) V+\left[\begin{array}{c}
u \\
\mathcal{P}_{L}
\end{array}\right] \oslash V\right),
$$

where $C:=\operatorname{bdiag}\left\{C_{S}, C_{L}\right\}, G:=\operatorname{bdiag}\left\{G_{S}, G_{L}\right\}$ are matrices of appropriate dimensions. In the sequel we will employ the following definition.

Definition 2.1: A DC microgrid is a continuous-time system $\Sigma^{\tau}:=\left(\mathcal{V}, \mathcal{V}^{0}, \mathcal{U}, \mathcal{Y}, \mathrm{f}, \mathrm{u}, \mathrm{h}\right)$ where

- $\mathcal{V} \subset \mathbb{R}^{n}, \mathcal{V}^{0} \subset \mathcal{V}, \mathcal{U} \subset \mathbb{R}^{p}$ and $\mathcal{Y} \subset \mathbb{R}^{r}$, with $p \leq n$, are the set of state voltages, the set of initial state voltages, controllable input currents and measurable output voltages;

- $\mathrm{f}: \mathcal{V} \times \mathcal{U} \rightrightarrows \mathcal{V}$ is the continuous-time map associated to the dynamics (II.6);

- $\mathrm{u}: \mathcal{Y} \rightrightarrows \mathcal{U}$ is a sampled-data controller with a clock of period $\tau>0$.

- $\mathrm{h}: \mathcal{V} \rightarrow \mathcal{Y}$ is the continuous-time map associated to the measurable output voltages;

A trajectory of the system $\Sigma^{\tau}$ is a signal $V: \mathbb{T} \rightarrow \mathcal{V}$ where $V$ is continuous, $V(0) \in \mathcal{V}^{0}$ and for all $t>0, \dot{V} \in$ $\mathrm{f}\left(V(t), u_{k}\right)$, where $u_{k} \in \mathrm{u}(V(k \tau))$ for $t \in[k \tau,(k+1) \tau)$.

A straightforward property of the DC microgrid is that it is monotone - a fact that can be easily verified via the Kamke-Muller conditions for continuously differentiable vector fields, since we have:

$$
\frac{\partial \mathrm{f}_{i}}{\partial v_{j}} \geq 0, \quad \frac{\partial \mathrm{f}_{i}}{\partial p_{k}} \geq 0 \quad i, j \in \mathcal{N}, k \in \mathcal{N}_{L}, j \neq i .
$$

\section{PROBLEM FORMULATION}

As already mentioned, sources are usually equipped with a primary control layer that ensures the robustness of the grid in presence of sudden change and/or fluctuations in the load profiles. In order to formulate properly the control problem, we find convenient to define the following specification sets:

- the set of admissible state voltages

$$
\mathcal{V}_{\delta}:=\mathcal{I}_{\delta}\left(v^{\mathrm{nom}} 1_{n}\right) \subset \mathbb{R}^{n}, \quad v^{\mathrm{nom}}>0, \delta>0 ;
$$

- the set of admissible power injections $\mathcal{U}_{S}:=\mathcal{P}_{S}$

- the set of power sharing injections

$$
\mathcal{U}_{\Gamma}:=\left\{u \in \mathbb{R}^{m}: \Gamma u=1 \alpha\right\},
$$

with $\Gamma=\operatorname{diag}\left(\gamma_{j}\right) \in \mathbb{R}^{m \times m}$, where $\gamma_{j}>0, \alpha>0$.

The set $\mathcal{V}_{\delta}$ specifies that the system's trajectory must be kept sufficiently near the nominal value $v^{\text {nom }}>0$. The sets $\mathcal{U}_{S}$ and $\mathcal{U}_{\Gamma}$ account for the limited power available at each source and the verification of an appropriate power sharing. We are now ready to define the control problem.

Problem 3.1: Consider a DC microgrid $\Sigma^{\tau}$, with $\mathcal{V}=\mathcal{V}_{\delta}$, $\mathcal{U}=\mathcal{U}_{S} \cap \mathcal{U}_{\Gamma}$ and let $\mathcal{Y} \subset \mathbb{R}$ a set of measurable outputs.
Determine a sampled-data controller $\mathrm{u}: \mathcal{Y} \rightrightarrows \mathcal{U}$, with a clock period $\tau>0$, and a non-empty set $\mathcal{V}^{\star} \subseteq \mathcal{V}$ such that the closed-loop trajectories of the DC microgrid satisfy

$$
V(0) \in \mathcal{V}^{\star} \Rightarrow V(t) \in \mathcal{V}^{\star}, t>0 .
$$

The solution of the problem consists thus in determining an (output-based) safety controller that guarantees that the trajectories $(V(t), u(t))$ originating in the set $\mathcal{V}^{\star} \times \mathcal{U}$ remain there for any future time.

Remark 3.2: An important theoretical question is to determine an optimal (according to some cost function) tuple $\left(\mathcal{V}^{\star}, \mathcal{P}_{S}, \mathcal{P}_{L}\right)$ so that $\mathcal{V}^{\star}$ is invariant. This formulation scales to the traditional power flow optimization problem whenever we restrict the sets to be singletons.

\section{SYMBOLIC CONTROL}

We now illustrate how symbolic control techniques can be used to achieve the control objectives defined in Problem 3.1. For this purpose, we determine a symbolic model-termed abstraction - that is linked to the original system $\Sigma^{\tau}$ via an appropriate alternating simulation relation [10].

\section{A. Construction of an abstraction of the system}

An abstraction $\Sigma^{a}$ for the DC microgrid $\Sigma^{\tau}$ is a tuple $\Sigma^{\mathrm{a}}:=\left(\mathcal{V}^{\mathrm{a}}, \mathcal{U}^{\mathrm{a}}, \Delta\right)$, where $\mathcal{V}^{\mathrm{a}}$ and $\mathcal{U}^{\mathrm{a}}$ are finite (symbolic) sets of states and control inputs respectively, while $\Delta: \mathcal{V}^{\mathrm{a}} \times$ $\mathcal{U}^{\mathrm{a}} \rightrightarrows \mathcal{V}^{\mathrm{a}}$ is a nondeterministic transition relation. For the construction of the symbolic sets we proceed as follows. We discretize the state-space $\mathcal{V}$ of the original system into $N \geq 1$ intervals $q_{i}$ using a finite and uniform partition

$$
\mathcal{V}^{\mathrm{a}}:=\left\{q_{i} \mid i=1, \ldots, N\right\}
$$

and define the quantizer $Q: \mathcal{V} \rightarrow \mathcal{V}^{\mathrm{a}}$, so that $Q(V)=q$ if and only if $V \in q$. Moreover, we discretize the set of inputs $\mathcal{U}$ into $M \geq 2$ values $c_{h}$, with the discrete input set given by

$$
\mathcal{U}^{\mathrm{a}}:=\left\{c_{h} \mid h=1, \ldots, M\right\} .
$$

In order to characterize the transition relation, we recall that the reachable set of a DC microgrid $\Sigma^{\tau}$ at time $t>0$, from a set of states $\mathcal{V}_{x} \subseteq \mathcal{V}$, with a constant control input $u \in \mathcal{U}$, is given by:

$$
\mathfrak{R}_{t}\left(\mathcal{V}_{x}, u\right):=\left\{\Phi\left(t ; V_{0}, u\right): \mid V_{0} \in \mathcal{V}_{x}, u \in \mathcal{U}\right\}
$$

Hence, under the same conditions, the reachable set over $[0, T]$ reads $\mathfrak{R}_{[0, T]}:=\bigcup_{t \in[0, T]} \mathfrak{R}_{t}$.

Recalling that the primary controller to be designed is implemented by a microprocessor with a clock of period $\tau>0$, the transition relation $\Delta: \mathcal{V}^{\mathrm{a}} \times \mathcal{U}^{\mathrm{a}} \rightrightarrows \mathcal{V}^{\mathrm{a}}$ can be defined as follows. For any $q, q^{\prime} \in \mathcal{V}^{\mathrm{a}}, c \in \mathcal{U}^{\mathrm{a}}, q^{\prime} \in \Delta(q, c)$ if and only if :

$$
\mathfrak{R}_{[0, \tau]}(q, c) \subseteq \mathcal{V}, \quad q^{\prime} \cap \mathfrak{R}_{\tau}(q, c) \neq \emptyset .
$$

The problem of computing reachable sets has been largely investigated in literature-see for example [13], [14] and [15]. For the system under study, the monotonicity property 
established in Section II can be exploited for the construction of tight systems abstractions [16], [17].

\section{B. Controller synthesis}

Using the aforementioned construction, we next show that we can guarantee some suitable equivalence between the abstraction $\Sigma^{\mathrm{a}}$ and the original system $\Sigma^{\tau}$. We first introduce the following notation. For a state $q \in \mathcal{V}^{\mathrm{a}}$ of the abstraction, we denote the set of enabled inputs by $\operatorname{enab}^{\mathrm{a}}(q)=\{c \in$ $\left.\mathcal{U}^{\mathrm{a}} \mid \Delta(q, c) \neq \emptyset\right\}$ and denote the set of non-blocking states by $\mathrm{nb}^{\mathrm{a}}=\left\{q \in \mathcal{V}^{\mathrm{a}} \mid \operatorname{enab}^{\mathrm{a}}(q) \neq \emptyset\right\}$. We are now ready to present the following proposition. The proof, which is the same reported in [18], is here omitted for sake of brevity.

Proposition 4.1: Consider a continuous-time DC microgrid $\Sigma^{\tau}:=(\mathcal{V}, \mathcal{U}, \mathcal{Y}, \mathrm{f}, \mathrm{u}, \mathrm{h})$ and the correspondent abstraction $\Sigma^{\mathrm{a}}:=\left(\mathcal{V}^{\mathrm{a}}, \mathcal{U}^{\mathrm{a}}, \Delta\right)$ constructed as above. Let $V \in q$, $c \in \operatorname{enab}^{\mathrm{a}}(q)$, with $q \in \mathrm{nb}^{\mathrm{a}}$. Then, for any reached point $x \in \Phi(\tau ; V, c)$, there exists $q^{\prime} \in \Delta(q, c)$ such that $x \in q^{\prime}$.

The abstraction $\Sigma^{\mathrm{a}}$ is then related to the uncontrolled dynamics of $\Sigma^{\tau}$ (i.e. with $\mathrm{u}(y)=\mathcal{U}$ for all $y \in \mathcal{Y}$ ), via an alternating simulation relation at sampling instants. We are now ready for the synthesis of a controller that solves Problem 3.1. For illustrative purposes, we first consider the centralized full information case (i.e. with $\mathcal{Y}=\mathcal{V}_{\delta}$ ). The problem of partial information case is instead postponed to Section V.

Using a safety game [10], we thus synthesize a symbolic controller $\mathrm{c}: \mathcal{V}^{\mathrm{a}} \rightrightarrows \mathcal{U}^{\mathrm{a}}$ for the abstraction that guarantees that all trajectories of the abstraction originating in some $\mathcal{V}^{\mathrm{a} \star} \subseteq \mathcal{V}^{\mathrm{a}}$, remain there for any future time. Therefore, the formal relation between the original system $\Sigma$ and its abstraction $\Sigma^{\mathrm{a}}$ can be used to refine the symbolic controller into a sampled-data controller $\mathrm{u}: \mathcal{V}_{\delta} \rightrightarrows \mathcal{U}$ for the original system. More precisely, recalling the definition of the quantizer we have $\mathrm{u}(V)=\mathrm{c}(Q(V))$. Since any enabled control input belongs to $\mathcal{U}=\mathcal{U}_{S} \cap \mathcal{U}_{\Gamma}$ by construction, the controller $\mathrm{u}(V)$ ensures that the control objectives defined in Problem 3.1 are achieved at all sampling instants. Guarantees on the intersample behaviour are, on the other hand, provided by the way the abstraction has been constructed. More precisely, by condition (IV.1) that ensures that all trajectories of the system remains in $\mathcal{V}_{\delta}$ between consecutive sampling instants.

\section{Contract-BASED Design}

In general, loads may be not equipped with measurement units that broadcast information on the local voltages to the controller. Hence, we here consider the Problem 3.1 by assuming that the voltages associated to the sources are the only available measurements. Despite this limitation, sources are allowed to communicate and the controller is referred as centralized under partial information.

\section{A. System decomposition}

Given this scenario, we find convenient to decompose the DC microgrid $\Sigma^{\tau}$ into two components, a source system $\Sigma_{S}^{\tau}$ and a load system $\Sigma_{L}$, which are defined as follows.
A source subsystem of a DC microgrid $\Sigma^{\tau}$ is a system $\Sigma_{S}^{\tau}:=\left(\mathcal{V}_{S}, \mathcal{V}_{S}^{0}, \mathcal{U}, \mathcal{W}_{S}, \mathrm{f}_{S}, \mathrm{u}\right)$ where:

- $\mathcal{V}_{S} \subset \mathbb{R}^{m}, \mathcal{V}_{S}^{0} \subset \mathcal{V}_{S}, \mathcal{U} \subset \mathbb{R}^{p}$ and $\mathcal{W}_{S} \subset \mathbb{R}^{n-m}$ are the set of measurable state voltages, initial state voltages, controllable power inputs and voltage perturbations respectively;

- $\mathrm{f}_{S}: \mathcal{V}_{S} \times \mathcal{U} \times \mathcal{W}_{S} \rightrightarrows \mathcal{V}_{S}$ is the continuous-time map:

$$
\mathrm{f}_{S}:=C_{S}^{-1}\left[-\left(G_{S}+\mathcal{L}_{S}\right) V_{S}-\mathcal{L}_{m} w_{S}+u \oslash V_{S}\right]
$$

- $\mathrm{u}: \mathcal{V}_{S} \rightrightarrows \mathcal{U}$ is a sampled-data controller with a clock of period $\tau>0$ (to be designed).

A trajectory of the subsystem $\Sigma_{S}^{\tau}$ is a tuple $\left(V_{S}, w_{S}\right)$ : $\mathbb{T} \rightarrow \mathcal{V}_{S} \times \mathcal{W}_{S}$ where $V_{S}$ and $w_{S}$ are continuous, $V_{S}(0) \in$ $\mathcal{V}_{S}^{0}$ and for all $t>0, \dot{V}_{S}=\mathrm{f}_{S}\left(V_{S}(t), w_{S}(t), u_{k}\right)$, where $u_{k} \in \mathrm{u}\left(V_{S}(k \tau)\right)$ for $t \in[k \tau,(k+1) \tau)$.

A load subsystem of a DC microgrid $\Sigma^{\tau}$ is a system $\Sigma_{L}:=$ $\left(\mathcal{V}_{L}, \mathcal{V}_{L}^{0}, \mathcal{W}_{L}, f_{L}\right)$ where:

- $\mathcal{V}_{L} \subset \mathbb{R}^{m}, \mathcal{V}_{L}^{0} \subset \mathcal{V}_{L}$ and $\mathcal{W}_{L} \subset \mathbb{R}^{n-m}$ are the set of state voltages, initial state voltages and voltage perturbations respectively;

- $\mathrm{f}_{L}: \mathcal{V}_{L} \times \mathcal{W}_{L} \rightrightarrows \mathcal{V}_{L}$ is the continuous-time map:

$$
\mathrm{f}_{L}:=C_{L}^{-1}\left[-\left(G_{L}+\mathcal{L}_{L}\right) V_{L}-\mathcal{L}_{m}^{\top} w_{L}+\mathcal{P}_{L} \oslash V_{L}\right] .
$$

A trajectory of the subsystem $\Sigma_{L}$ is a tuple $\left(V_{L}, w_{L}\right)$ : $\mathbb{T} \rightarrow \mathcal{V}_{L} \times \mathcal{W}_{L}$ where $V_{L}$ and $w_{L}$ are continuous, $V_{L}(0) \in \mathcal{V}_{L}^{0}$ and for all $t>0, \dot{V}_{L}=\mathrm{f}_{L}\left(V_{L}(t), w_{L}(t)\right)$.

Since measurements are available only at the source subsystem, the Problem 3.1 must be now solved for the case $\mathcal{Y}=\mathcal{V}_{S}$ and abstractions for both subsystems can be constructed as detailed in the previous section.

Remark 5.1: It is immediate to see that the composition of the systems $\Sigma_{S}, \Sigma_{L}$ via the interconnection laws $w_{S}=V_{L}$, $w_{L}=V_{S}$ allows to recover the system $\Sigma^{\tau}$.

\section{B. Compositional reasoning}

In order to deal with safety synthesis under partial information, we use the notion of continuous-time assume guarantee contracts [19].

Definition 5.2: . Let $\Sigma=\left(\mathcal{V}, \mathcal{V}^{0}, \mathcal{U}, \mathcal{W}, \mathrm{f}\right)$, be a continuous-time system where $\mathcal{V} \subset \mathbb{R}^{n}$ is the set of states, $\mathcal{V}^{0} \subseteq \mathcal{V}$ is the set of initial states, $\mathcal{U} \subset \mathbb{R}^{m}$ is the set of control inputs, $\mathcal{W} \subset \mathbb{R}^{p}$ is the set of perturbations and $\mathrm{f}$ is a locally Lipschitz continuous-time map associated to the dynamics $\dot{V} \in \mathrm{f}(V, \mathcal{W}, u)$. A continuous-time assumeguarantee contract for the system $\Sigma$ is a pair $\mathcal{C}=(A, G)$ where:

- $A \subseteq \mathcal{W}$ is the set of assumptions;

- $G \subseteq \mathcal{V}$, is a nonempty set of guarantees where $G$ is closed.

We say that $\Sigma$ satisfies a contract $\mathcal{C}=(A, G)$, denoted $\Sigma \models \mathcal{C}$, if for any initial condition $V(0) \in G$ and any trajectory $(V, w): \mathbb{T} \rightarrow \mathcal{V} \times \mathcal{W}$ of the system $\Sigma$ originating in $V(0)$, the following logical implication is satisfied:

for all $T \in \mathbb{R}$, such that for all $\left.t \in[0, T], w_{(} t\right) \in A$, we have the existence of $\epsilon>0$, such that for all $t \in[0, T+\epsilon]$, $V(t) \in G$. 
We can now use the results on compositional reasoning of assume-guarantee contracts of [19, Th. 1-2] to obtain the following proposition.

Proposition 5.3 ( [19]): Let $\mathcal{C}_{i}=\left(A_{i}, G_{i}\right)$ be continuoustime assume-guarantee contracts for the systems $\Sigma_{i}, i \in$ $\{S, L\}$ and assume that $\Sigma_{S} \models \mathcal{C}_{S}, \Sigma_{L} \models \mathcal{C}_{L}$. Then, if

(i) $G_{L} \times G_{S} \subseteq A_{S} \times A_{L}$;

(ii) $G_{S} \times G_{L} \subseteq \mathcal{V}_{S} \times \mathcal{V}_{L}$

the control objective defined in Problem 3.1 is achieved with $\mathcal{V}^{\star}=G_{S} \times G_{L}$.

To ensure that a component satisfies its contract, we can rely on symbolic methods, similarly to [18]. For systems with no control input, as the load subsystem, the same approach can be used to verify whether the contract is naturally satisfied or not. Nevertheless, the choice of appropriate contracts is not trivial. In fact, Proposition 5.3 merely states that if we are able to construct suitable contracts $\mathcal{C}_{S}$ and $\mathcal{C}_{L}$ for the subsystems $\Sigma_{S}$ and $\Sigma_{L}$, then Problem 3.1 is solved for some $\mathcal{V}^{\star} \subseteq \mathcal{V}$. However, no constructive procedure is provided for the derivation of such contracts.

\section{Parametric contracts synthesis}

In order to explore systematically the space of feasible contracts for a system $\Sigma$, we here consider families of contracts $\mathcal{C}(\alpha, \gamma)$ as assumption-guarantee pairs parameterized by the parameters $(\alpha, \gamma) \in \mathbb{R}^{a} \times \mathbb{R}^{g}$, where $a, g$ are positive integers. The use of parametric assume-guarantee contracts has been already suggested in the context of vehicular traffic networks [20]. For an appropriate formalization, we introduce the following definition.

Definition 5.4: Consider a family of continuous-time assume-guarantee contracts $\mathcal{C}(\alpha, \gamma)=(A(\alpha), G(\gamma))$ for the system $\Sigma$, parametrized by $A: \mathbb{R}^{a} \rightarrow 2^{\mathcal{W}}, G: \mathbb{R}^{g} \rightarrow 2^{\mathcal{V}}$, with $a, g$ positive integers. Then $\mathcal{C}(\alpha, \gamma)$ is said to be satisfied by $\Sigma$ on $\mathrm{F} \subseteq 2^{\mathcal{W}} \times 2^{\mathcal{V}}$ if it is satisfied by $\Sigma$ for any $(\alpha, \gamma) \in \mathrm{F}$. The maximal region where $\mathcal{C}(\alpha, \gamma)$ is satisfied by $\Sigma$ is called the feasibility region of $\Sigma$ with respect to $\mathcal{C}$.

The set $\mathrm{F}$ determines, on the space of parameters, a family of contracts that are satisfied by the system. For the general case the computation of the feasibility region is far from being obvious. However, for the present case, monotonicity of the contracts can be exploited for an efficient calculation of a lower approximation of the feasibility region $\mathrm{F}$. This is illustrated in the following result.

Proposition 5.5: Consider a family of assume-guarantee contracts $\mathcal{C}(\alpha, \gamma)$ for the system $\Sigma$, where $(\alpha, \gamma) \in 2^{\mathcal{W}} \times 2^{\mathcal{V}}$. If, for any $\alpha, \alpha^{\prime} \in 2^{\mathcal{W}}, \gamma, \gamma^{\prime} \in 2^{\mathcal{V}}$, the following logical implications are satisfied:

$$
\alpha \leq \alpha^{\prime} \Rightarrow A(\alpha) \subseteq A\left(\alpha^{\prime}\right), \quad \gamma \leq \gamma^{\prime} \Rightarrow G(\gamma) \subseteq G\left(\gamma^{\prime}\right)
$$

then the following property holds:

$$
\left.((\alpha, \gamma) \in \mathrm{F}) \wedge\left(\alpha^{\prime} \leq \alpha\right) \wedge\left(\gamma^{\prime} \geq \gamma\right)\right) \Rightarrow\left(\left(\alpha^{\prime}, \gamma^{\prime}\right) \in \mathrm{F}\right) .
$$

The proposition implies that the boundary of the feasibility region $\mathrm{F}$ has the structure of a Pareto front and can therefore be approximated arbitrarily close, from inside and outside, adapting efficient multidimensional binary search algorithms used in multi-objective optimization [21], [22]. A similar approach for the computation of the feasibility region was applied to timing contracts in [23].

We are now ready to write a corollary of Proposition 5.3 that extends the result to all possible contracts defined on the space of parameters. The result is straightforward and then stated without proof.

Corollary 5.6: Let $\mathcal{C}_{i}\left(\alpha_{i}, \gamma_{i}\right)=\left(A_{i}\left(\alpha_{i}\right), G_{i}\left(\gamma_{i}\right)\right)$ be a family of continuous-time assume-guarantee contracts for $\Sigma_{i}$, where $\left(\alpha_{i}, \gamma_{i}\right) \in 2^{\mathcal{W}_{i}} \times 2^{\mathcal{V}_{i}}, i \in\{S, L\}$ and defined as follows:

$$
\begin{aligned}
& \mathcal{V}_{S}=\bigcup_{\alpha_{L} \in 2^{\mathcal{W}_{S}}} A_{L}\left(\alpha_{L}\right)=\bigcup_{\gamma_{S} \in 2^{\mathcal{V}_{S}}} G_{S}\left(\gamma_{S}\right) \\
& \mathcal{V}_{L}=\bigcup_{\alpha_{S} \in 2^{\mathcal{W}_{L}}} A_{S}\left(\alpha_{S}\right)=\bigcup_{\gamma_{L} \in 2^{\mathcal{V}_{L}}} G_{L}\left(\gamma_{L}\right) .
\end{aligned}
$$

Assume that there exist non-empty sets $\mathrm{F}_{S}, \mathrm{~F}_{L}$ such that $\Sigma_{S} \models \mathcal{C}_{S}\left(\alpha_{S}, \gamma_{S}\right)$ on $\mathrm{F}_{S}$ and $\Sigma_{L} \models \mathcal{C}_{L}\left(\alpha_{L}, \gamma_{L}\right)$ on $\mathrm{F}_{L}$. Then, for any $\left(\alpha_{S}, \gamma_{S}\right) \in \mathrm{F}_{S},\left(\alpha_{L}, \gamma_{L}\right) \in \mathrm{F}_{L}$ such that $G_{L}\left(\gamma_{L}\right) \times G_{S}\left(\gamma_{S}\right) \subseteq A_{S}\left(\alpha_{S}\right) \times A_{L}\left(\alpha_{L}\right)$, the control objective defined in Problem 3.1 is achieved with $\mathcal{V}^{\star}\left(\gamma_{S}, \gamma_{L}\right)=G_{S}\left(\gamma_{S}\right) \times G_{L}\left(\gamma_{L}\right)$

\section{NUMERICAL EXAMPLES}

In this section, we show the practicality of our approach with the following two examples.

\section{A. Two-units case}

We consider a two-units source-load DC microgrid with the following parameters: $C_{S}=2.2 \mathrm{mF}, C_{L}=1.8 \mathrm{mF}$ the units capacitances; $G_{S}=G_{L}=0.025 \Omega^{-1}$ the units conductances; $G_{T}=6.65 \Omega^{-1}$ the line conductance. The system is supposed to operate within a region $\mathcal{V}_{\delta}$, with grid nominal voltage $v^{\text {nom }}=450 \mathrm{~V}$ and $\delta=0.01 \cdot v^{\text {nom }}$, which corresponds to a maximal $1 \%$ deviation from the grid nominal value. Following the approach presented in Section V, the DC microgrid is decomposed into a source subsystem $\Sigma_{S}$ and a load subsystem $\Sigma_{L}$ and two families of assumeguarantee contracts $\mathcal{C}_{i}\left(\alpha_{i}, \gamma_{i}\right)$ are considered, $i=\{S, L\}$. Such families are locally constructed over the same space $[-2 \delta, 0] 1_{2}$, in a way that the assumptions and guarantees sets read respectively:

$$
\begin{aligned}
& A_{i}\left(\alpha_{i}\right)=\left[v^{\mathrm{nom}}-\delta-\alpha_{i, 1}, v^{\mathrm{nom}}+\delta+\alpha_{i, 2}\right], \\
& G_{i}\left(\gamma_{i}\right)=\left[v^{\mathrm{nom}}-\delta-\gamma_{i, 1}, v^{\mathrm{nom}}+\delta+\gamma_{i, 2}\right] .
\end{aligned}
$$

Assumptions parameters can be then combined with guarantee parameters via the sources-loads interconnection law, see also Remark 5.1, by taking $\alpha_{S, 1}=\gamma_{L, 1}, \quad \alpha_{S, 2}=\gamma_{L, 2}$. It can be easily checked then that the proposed families of contract verify conditions (V.1) of Proposition 5.5 and then the feasibility regions $\mathrm{F}_{i}$ have the structure of Pareto fronts. Hence, the global feasible region can be obtained by intersecting such regions. For illustrative purposes, we select $\alpha_{S, 2}=\gamma_{S, 2}=\alpha_{L, 2}=\gamma_{L, 2}=0$, which allows for the representation of the space of parameters on a two-dimensional 


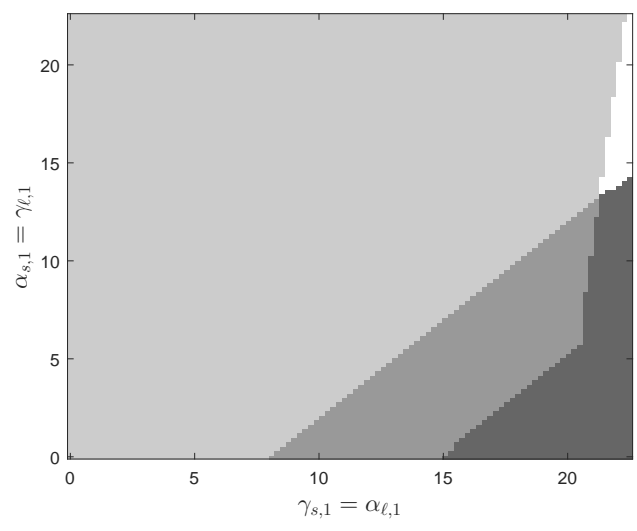

Fig. 1: Global feasibility region (medium gray) obtained as the intersection of the source (light gray) and load (dark gray) feasibility regions.

plane, as shown in Fig. 1. To compute the feasibility regions, we used a symbolic approach, with sampling period for the abstractions $\tau=0.1 \mathrm{~ms}$, which corresponds to the clock of the sampled-data controller to be designed. Discretization parameters are $N=10$, denoting the dimension of the (symbolic) state-space, and $M=20$ denoting the number of discrete inputs. The region $\mathrm{F}_{L}$ associated to the load subsystem can be computed by checking the satisfaction of the correspondent contract $\mathcal{C}_{L}\left(\alpha_{L}, \gamma_{L}\right)$, while for the source subsystem we take advantage of the additional degree of freedom provided by the control input to construct a sampled-data controller $u: \mathcal{Y} \rightrightarrows \mathcal{U}$ that enforces the satisfaction of the contract $\mathcal{C}_{S}\left(\alpha_{S}, \gamma_{S}\right)$. For the design, we select thus a feasible assume-guarantee pair that maximize the domain of the controller. Responses to different, bounded, time-varying power demands are illustrated in Fig. 2.

\section{B. Four-units case}

We consider a four-terminal DC microgrid as the one depicted in Fig. 3. We assume that two units, namely Unit 2 and 3 , are equipped with a primary control layer, while the remaining two units, Unit 1 and Unit 4 correspond to loads with demand varying steadily around a constant power reference. Bus and network parameters are provided in Table I and Table II respectively.

TABLE I: Bus parameters.

\begin{tabular}{ccccc}
\hline & 1 & 2 & 3 & 4 \\
\hline$C_{i}(\mathrm{mF})$ & 2.2 & 1.9 & 1.5 & 1.7 \\
\hline
\end{tabular}

TABLE II: Network parameters in $\Omega^{-1}$.

\begin{tabular}{llllll}
\hline$G_{12}$ & 5.215 & $G_{13}$ & 4.615 & $G_{14}$ & 4.515 \\
$G_{24}$ & 6.015 & $G_{34}$ & 5.615 & & \\
\hline
\end{tabular}

The system is supposed to operate within a region $\mathcal{V}_{\delta}$, with grid nominal voltage $v^{\text {nom }}=450 \mathrm{~V}$ and $\delta=0.005$. The DC microgrid is then decomposed into two-dimensional source
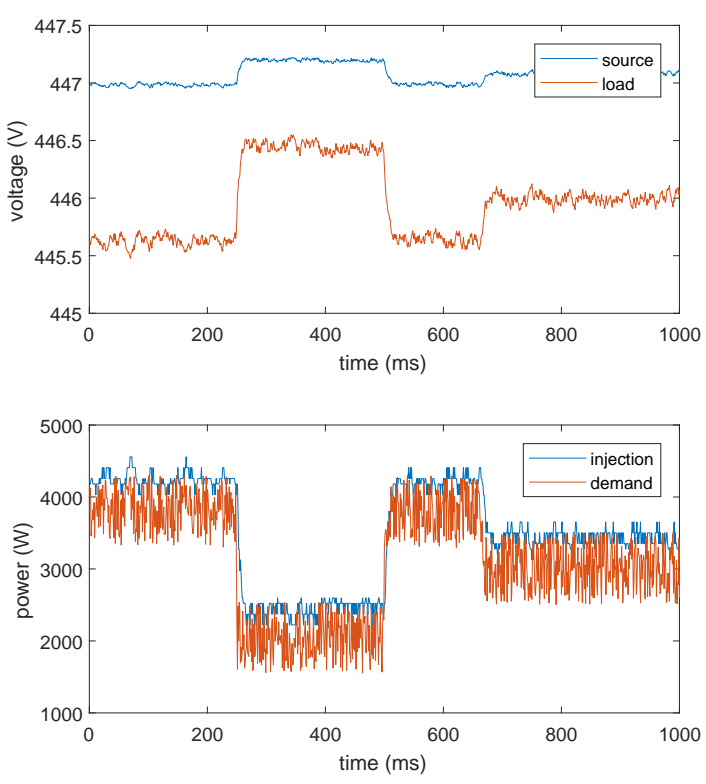

Fig. 2: Voltage and power responses to different power demands, using the proposed sampled-data controller.

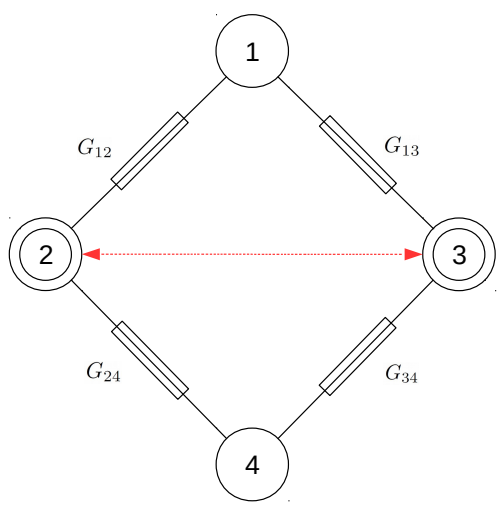

Fig. 3: The four-units architecture used for the simulations. Circles correspond to units, with sources denoted by double circles. Solid lines denote the transmission lines and dashed blue arrows represent the communication between units.

and load subsystems $\Sigma_{S}$ and $\Sigma_{L}$. Symbolic abstractions are thus constructed using the same discretization and two families of assume-guarantee contracts $\mathcal{C}_{i}\left(\alpha_{i}, \gamma_{i}\right)$ are considered, $i=\{S, L\}$. To validate our controller, we assume that the load power demands for Unit 1 and Unit 4 are as follows. Unit 1 is demanding $1 \mathrm{~kW}$ from 0 to $250 \mathrm{~ms}$, immediately after stepping up to $5 \mathrm{~kW}$. Unit 4 on the other hand is supposed to be characterized by a constant demand of $0.5 \mathrm{~kW}$ from 0 to $750 \mathrm{~ms}$, then stepping up to $4.5 \mathrm{~kW}$. Both demands are affected by small noise. Source power injections are both limited at $12 \mathrm{~kW}$. The controller is implemented via a microprocessor of clock period $\tau=0.1 \mathrm{~ms}$ and verifies 
the power sharing property with ratio $\Gamma=\mathbb{I}_{2}$ (equal power distribution) by construction. Among the feasible controllers, the one that minimizes the quadratic injection is selected. Power injections and voltage responses are illustrated in Fig. 4. As expected, the controller guarantees that voltages are kept sufficiently near the nominal value and that the power injection is shared in accordance with the assigned ratio.
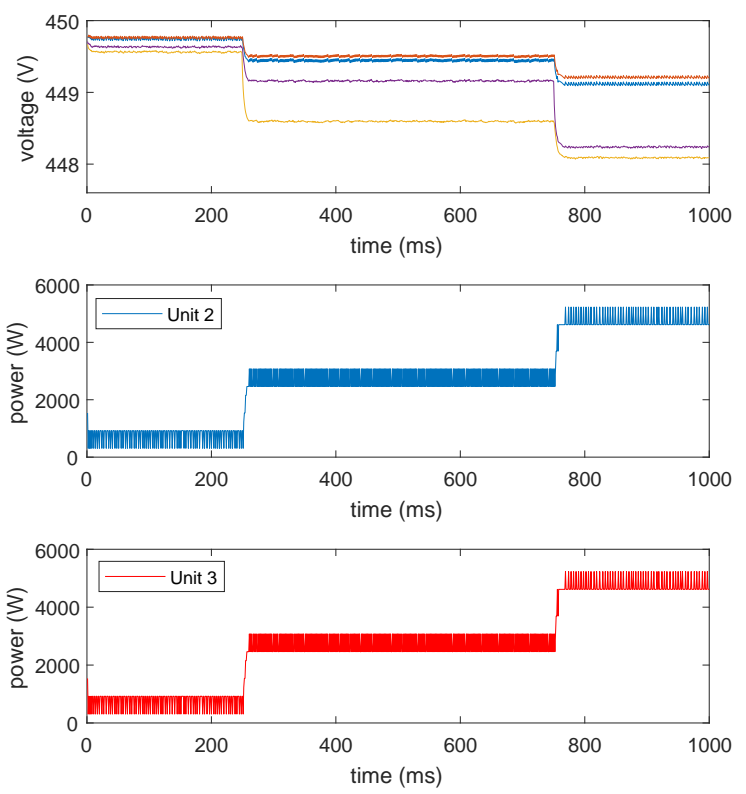

Fig. 4: Voltage and power responses to different power demands, using the proposed sampled-data controller.

\section{CONCLUSIONS}

In this work we addressed the problem of designing centralized primary controllers for nonlinear time-varying DC microgrids using symbolic methods, considering both the full information and partial information case. In the former, a traditional approach is employed to construct the system's abstraction, from which a safety controller is easily derived and then refined into a sampled-data controller for the original system. For the partial information case instead, we defined an appropriate system's decomposition, for each of the components an abstraction is derived and a family of locally satisfied, assume-guarantee contracts is constructed. Therefore, locally feasible contracts are combined to establish families of contracts satisfied by the overall system, which are then used for the design of the controllers, similarly to the full information case.

These contributions should be intended as a preliminary step towards the development of a more general theory for contract-based design of symbolic controllers for DC microgrids. Further investigation will focus on the use of parametric contracts in two main directions. First, establish a procedure for optimal selection of performance-guranteed controllers. Second, extend the design to the case of distributed and decentralized communication.

\section{REFERENCES}

[1] T. Dragičević, X. Lu, J. C. Vasquez, and J. M. Guerrero, "Dc microgrids-part ii: A review of power architectures, applications, and standardization issues," IEEE Transactions on Power Electronics, vol. 31, pp. 3528-3549, May 2016.

[2] T. Dragičević, X. Lu, J. C. Vasquez, and J. M. Guerrero, "Dc microgrids-part i: A review of control strategies and stabilization techniques," IEEE Transactions on Power Electronics, vol. 31, pp. 48764891, July 2016.

[3] F. Dörfler, J. W. Simpson-Porco, and F. Bullo, "Electrical networks and algebraic graph theory: Models, properties, and applications," Proceedings of the IEEE, vol. 106, pp. 977-1005, May 2018.

[4] A. S. Matveev, J. E. Machado, R. Ortega, J. Schiffer, and A. Pyrkin, "On the existence and long-term stability of voltage equilibria in power systems with constant power loads," arXiv preprint arXiv:1809.08127, 2018.

[5] J. Liu, W. Zhang, and G. Rizzoni, "Robust stability analysis of dc microgrids with constant power loads," IEEE Transactions on Power Systems, vol. 33, no. 1, pp. 851-860, 2018.

[6] C. D. Persis, E. R. Weitenberg, and F. Dörfler, "A power consensus algorithm for dc microgrids," Automatica, vol. 89, pp. 364 - 375, 2018.

[7] J. Zhao and F. Dörfler, "Distributed control and optimization in DC microgrids," Automatica, vol. 61, pp. 18 - 26, 2015.

[8] M. Cucuzzella, S. Trip, C. D. Persis, X. Cheng, A. Ferrara, and A. van der Schaft, "A robust consensus algorithm for current sharing and voltage regulation in dc microgrids," IEEE Transactions on Control Systems Technology, pp. 1-13, 2018.

[9] P. Nahata, R. Soloperto, M. Tucci, A. Martinelli, and G. FerrariTrecate, "A passivity-based approach to voltage stabilization in $\mathrm{dc}$ microgrids with zip loads," tech. rep., 2018.

[10] P. Tabuada, Verification and control of hybrid systems: a symbolic approach. Springer Science \& Business Media, 2009.

[11] C. Belta, B. Yordanov, and E. Gol, Formal methods for discrete-time dynamical systems. Springer, 2017.

[12] C. Baier and J.-P. Katoen, Principles of model checking. MIT press, 2008.

[13] N. Ramdani, N. Meslem, and Y. Candau, "Computing reachable sets for uncertain nonlinear monotone systems," Nonlinear Analysis: Hybrid Systems, vol. 4, no. 2, pp. 263-278, 2010.

[14] C. Le Guernic and A. Girard, "Reachability analysis of linear systems using support functions," Nonlinear Analysis: Hybrid Systems, vol. 4, no. 2, pp. 250-262, 2010.

[15] G. Reissig, A. Weber, and M. Rungger, "Feedback refinement relations for the synthesis of symbolic controllers," IEEE Transactions on Automatic Control, vol. 62, no. 4, pp. 1781-1796, 2017.

[16] S. Coogan and M. Arcak, "Finite abstraction of mixed monotone systems with discrete and continuous inputs," Nonlinear Analysis: Hybrid Systems, vol. 23, pp. 254-271, 2017.

[17] P.-J. Meyer, A. Girard, and E. Witrant, "Safety control with performance guarantees of cooperative systems using compositional abstractions," in Proceedings of the $5^{\text {th }}$ IFAC Conference on Analysis and Design of Hyrbid Systems, pp. 317-322, 2015.

[18] A. Saoud, A. Girard, and L. Fribourg, "Contract based design of symbolic controllers for interconnected multiperiodic sampled-data systems," in 2018 IEEE Conference on Decision and Control (CDC), pp. 773-779, IEEE, 2018.

[19] A. Saoud, A. Girard, and L. Fribourg, "On the composition of discrete and continuous-time assume-guarantee contracts for invariance," in 2018 European Control Conference (ECC), pp. 435-440, IEEE, 2018.

[20] E. Kim, M. Arcak, and S. Seshia, "Compositional controller synthesis for vehicular traffic networks," in IEEE Conference on Decision and Control, pp. 6165-6171, 2015.

[21] J. Legriel, C. Le Guernic, S. Cotton, and O. Maler, "Approximating the pareto front of multi-criteria optimization problems," in International Conference on Tools and Algorithms for the Construction and Analysis of Systems, pp. 69-83, Springer, 2010.

[22] P. Tendulkar, Mapping and scheduling on multi-core processors using SMT solvers. PhD thesis, Universite de Grenoble I-Joseph Fourier, 2014.

[23] M. Al Khatib, A. Girard, and T. Dang, "Scheduling of embedded controllers under timing contracts," in Proceedings of the 20th International Conference on Hybrid Systems: Computation and Control, pp. 131-140, ACM, 2017. 